\title{
The hypoglycaemic action of metformin
}

\author{
K. N. Frayn \\ B.A.
}

Research Assistant

\author{
P. I. AdNITT \\ M.D., M.R.C.P.
}

Senior Registrar in Medicine

\author{
PAul Turner \\ M.D., B.Sc., M.R.C.P. \\ Reader in Clinical Pharmacology \\ The Diabetic Clinic and Department of Clinical Pharmacology, \\ St Bartholomew's Hospital, London, E.C.1
}

\begin{abstract}
Summary
Twenty-four-hour excretion of D-xylose after oral administration in nine diabetic patients, and of 3-0methyl-D-glucose in six patients, showed no significant change after treatment with metformin. There was, however, a significant reduction in mean fasting blood glucose levels in thirteen patients from 160 to $115 \mathrm{mg} /$ $100 \mathrm{ml}(P<0.001)$, and the intravenous glucose tolerance improved in twelve of these patients $(P<0.01)$, during treatment with metformin.

It is concluded that the hypoglycaemic action of metformin does not depend primarily on an intestinal effect.
\end{abstract}

\section{Introduction}

It has recently been suggested that treatment of diabetic patients with sulphonylureas may be associated with an increased mortality from cardiovascular disease (University Group Diabetes Program, 1970). The only other satisfactory oral antidiabetic agents available at the present time are the diguanides. The mode of action of the diguanides remains uncertain, although their effectiveness in controlling maturity-onset diabetes mellitus and in promoting weight loss in obese diabetic patients is well documented (Hooper, 1967; Clarke \& Duncan, 1968).

Czyzyk and co-workers (Czyzyk et al., 1968) found that treatment with phenformin caused an improvement in the oral glucose tolerance of diabetic patients but had no effect on the intravenous (i.v.) glucose tolerance. They concluded that phenformin decreases intestinal absorption of glucose. They were uncertain, however, to what extent this effect contributes to the therapeutic action of phenformin. Other workers have also reported that diguanides reduce intestinal absorption of glucose (Mainguet et al., 1970) and may also impair xylose absorption (Berchtold et al., 1969) in diabetic patients.

The present study was carried out to determine the extent to which the hypoglycaemic action of metformin is dependent on glucose malabsorption.

\section{Patients and methods}

D-Xylose and 3-0-methyl-D-glucose (3OMG) absorption tests were performed in obese maturityonset diabetic patients aged 55-66 years before and after treatment with metformin $1.5-3.0 \mathrm{~g}$ daily in divided doses for between 1 and 6 months. D-Xylose $25 \mathrm{~g}$ (nine patients) or 3OMG $10 \mathrm{~g}$ (six patients) in water was administered orally after an overnight fast. No food was allowed for the next $5 \mathrm{hr}$. Urine was collected for $24 \mathrm{hr}$ for estimation of xylose or 3OMG.

Blood glucose levels were measured and i.v. glucose tolerance tests performed after an overnight fast in a further thirteen overweight diabetic patients aged 29-73 years who had previously been treated by diet alone. These measurements were repeated after 1-2 weeks' treatment with metformin 1.5-3.0 g daily in divided doses. The i.v. glucose tolerance tests were carried out using D-glucose $25 \mathrm{~g}$ in $50 \%$ solution administered rapidly i.v. The rate constant for disappearance of glucose from blood ( $K$ value) was calculated over the next hour (Conard et al., 1953).

The patients undergoing xylose and 30MG tests were $30 \pm 13 \%$ over weight (mean \pm SD) and patients undergoing IVGTTs were $24 \pm 12 \%$ over weight (mean $\pm \mathrm{SD}$ ) (Metropolitan Life Insurance Company's figures).

D-Xylose was estimated by the method of Goodhart \& Kingston (1969), 3OMG by the method of Frayn \& Adnitt (1971), and D-glucose by the method of Morley, Dawson \& Marks (1968).

\section{Results \\ Xylose and 3OMG absorption (Fig. 1)}

Twenty-four-hour excretion of each sugar is expressed as a percentage of the administered dose. Mean xylose excretion before treatment was $39 \cdot 7 \%$ and after treatment $38.8 \%$ (mean difference \pm SE $-0.9 \pm 4.0 \%$ ). Mean 3OMG excretion before treatment was $82.5 \%$ and after treatment $84.9 \%$ (mean 


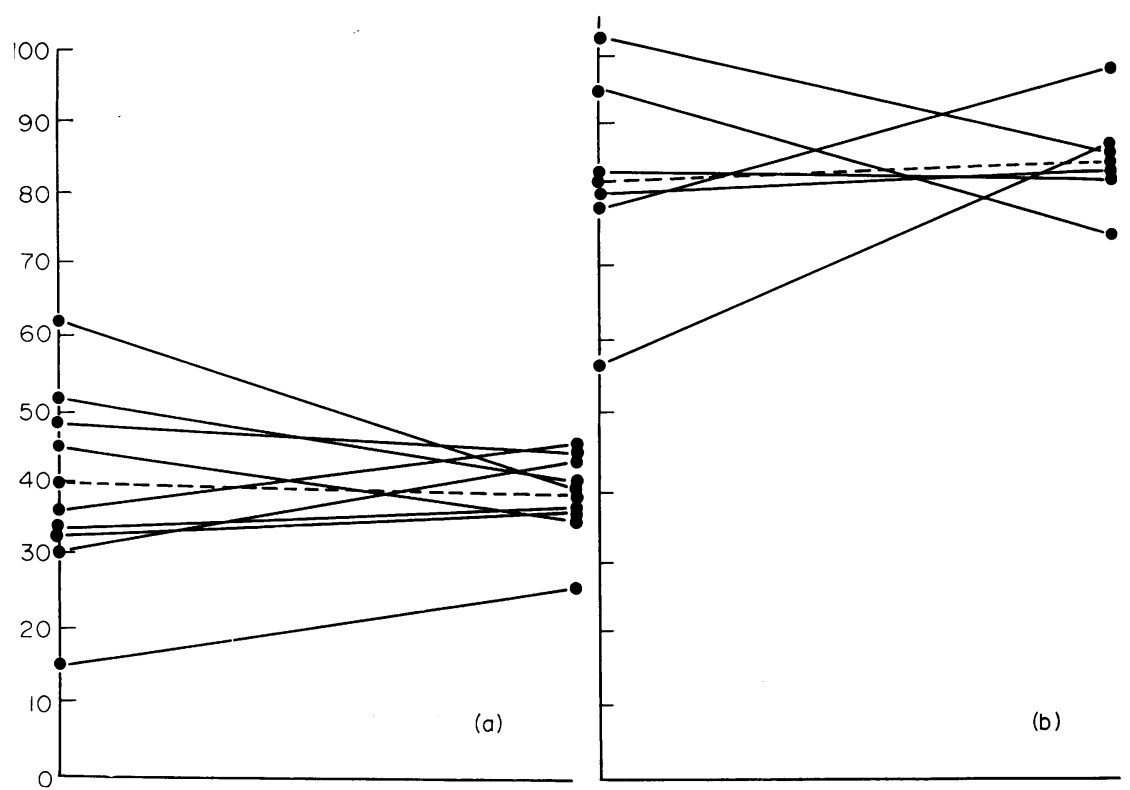

FIG. 1. Percentage excretion in $24 \mathrm{hr}$ following (a) $25 \mathrm{~g}$ xylose or (b) $10 \mathrm{~g}$ 3-0-methyl-Dglucose, orally before and after treatment with metformin. , Individual patients; -... - . mean.

difference $\pm \mathrm{SE}+2 \cdot 4 \pm 7 \cdot 9 \%$ ). There was no significant reduction in excretion of either sugar following metformin treatment.

\section{Fasting blood glucose levels (Fig. 2)}

Mean fasting blood glucose before treatment was $160 \mathrm{mg} / 100 \mathrm{ml}$ and after treatment $115 \mathrm{mg} / 100 \mathrm{ml}$ (mean difference $\pm \mathrm{SE}-45 \pm 9 \mathrm{mg} / 100 \mathrm{ml}$ ). This decrease was significant $(P<0 \cdot 001)$.

\section{Intravenous glucose tolerance tests (Fig. 3)}

Mean $K$ value before treatment was $0.59 \mathrm{~min}^{-1}$ and after treatment $0.82 \mathrm{~min}^{-1}$ (mean difference $\pm \mathrm{SE}+0.24 \pm 0.06, P<0.01)$. Intravenous glucose tolerance improved in twelve out of thirteen patients following metformin treatment. Figure 4 shows the change in $K$ value plotted against the initial $K$ value for each patient. There is no evidence that the improvement in i.v. glucose tolerance is related to the initial severity of the diabetes.

\section{Discussion}

Some (Berchtold et al., 1969; Stowers \& Bewsher, 1969) but not all (Creutzfeldt, 1969) workers have reported a significant reduction during diguanide treatment of the 5-hr xylose excretion following oral administration. Stowers \& Bewsher (1969), however, found no consistent reduction in the 24-hr xylose excretion. The present results show no reduction in xylose excretion over $24 \mathrm{hr}$. This suggests that although the diguanides may delay the excretion of xylose, there is no overall reduction in xylose absorption.

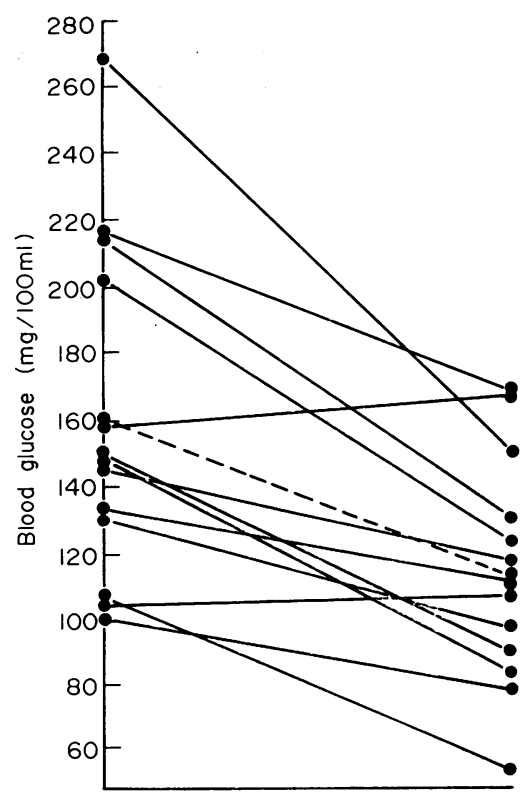

FIG. 2. Fasting blood glucose before and after metformin treatment. -.... .. mean. 


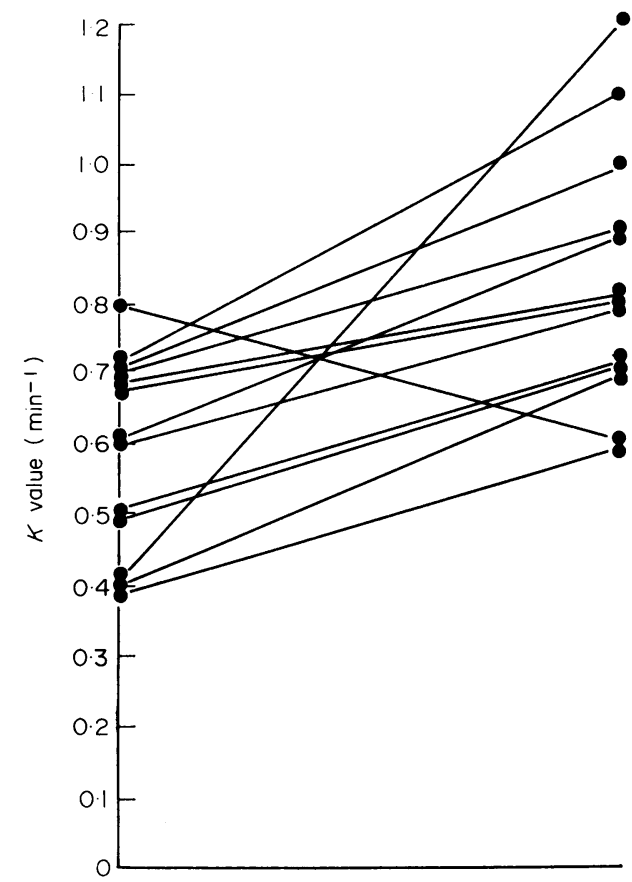

Fig. 3. Intravenous glucose tolerance $K$ values before and after metformin treatment.

3OMG excretion following oral administration may be used as an index of intestinal glucose absorption. It is absorbed by the same mechanism as glucose and is not significantly metabolized in man (Fordtran et al., 1962). We have found no reduction in $30 \mathrm{MG}$ excretion during the $24 \mathrm{hr}$ following oral administration in patients treated with metformin and therefore think it unlikely that metformin has an effect on intestinal glucose absorption.

The considerable improvement in i.v. glucose tolerance reported here is in contrast to the results of Czyzyk and co-workers (1968) who found no change in i.v. glucose tolerance in mild diabetic patients treated with phenformin for 3 days. There are several possible explanations for this discrepancy. In the present investigation the duration of treatment was longer. Our patients were also more severely diabetic; the mean initial $K$ value for the subjects in the study of Czyzyk was $0.8 \mathrm{~min}^{-1}$ whereas in the present study the mean initial $K$ value was $0.59 \mathrm{~min}^{-1}$ increasing to $0.82 \mathrm{~min}^{-1}$ after metformin treatment. This might suggest that the beneficial effect of diguanide treatment on intravenous glucose tolerance is related to the initial severity of the diabetes, especially since these drugs have no effect on the intravenous glucose tolerance of normal individuals (Hollobaugh, Rao \& Kruger, 1967, Lyngsøe \& Trap-Jensen, 1969). We have found no evidence that such a relationship exists (Fig. 4).

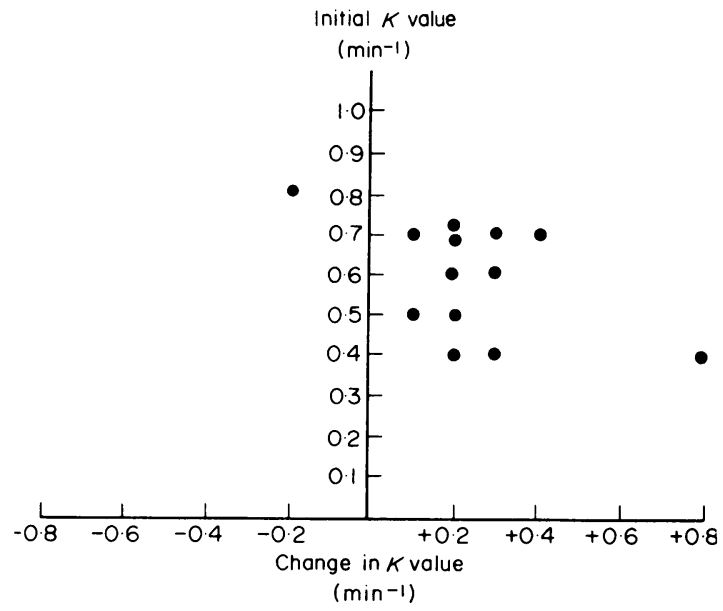

FIG. 4. Change in $K$ value following metformin treatment plotted against initial $K$ value for each patient.

The other possible explanation for the difference between the results presented here and those of Czyzyk is that metformin but not phenformin causes an improvement in intravenous glucose tolerance as has been suggested by Bouaziz (1966).

The improvement in intravenous glucose tolerance and reduction in fasting blood glucose level during metformin treatment cannot readily be explained on the basis of an intestinal effect on glucose absorption. Since we have also been unable to demonstrate any reduction in xylose or 3OMG absorption during metformin treatment, it would appear that the hypoglycaemic action of this drug does not depend primarily on an intestinal effect. There are, however, reports of interference with vitamin $B_{12}$ (Berchtold et al., 1969) and possibly folate absorption (Fearnley, 1969) during treatment with diguanides, and further tests of intestinal absorption are therefore being carried out.

\section{Acknowledgments}

We are grateful to Rona Laboratories Ltd and the Board of Governors, St Bartholomew's Hospital, for financial support. Dr K. O. Black kindly allowed us to study patients under his care.

\section{References}

Berchtold, P., Bolli, P., Arbenz, U. \& Keiser, G. (1969) Intestinale Absorptions störung infolge Metforminbehandlung (Zur Frage der Wirkungsweise der Biguanide). Diabetologia, 5, 405.

Bouaziz, P.J. (1966) Study of intravenously induced hyperglycaemia during antidiabetic therapy. Thèse Médicine, Paris.

Clarke, B.F. \& Duncan, L.J.P. (1968) Comparison of chlorpropamide and metformin treatment on weight and - blood-glucose response of uncontrolled obese diabetics. Lancet, i, 123. 
Conard, V., Franckson, J.R.M., Bastenie, P.A., Kestens, J. \& Kovacs, L. (1953) Etude critique du triangle d'hyperglycémie intraveineux chez l'homme normal et détermination d'un 'coefficient d'assimilation glucidique'. Archives internationales de Pharmacodynamie et de Thérapie, 93, 277.

Creutzfeldt, W. (1969) In discussion. Acta Diabetologica Latina, 6 Suppl. 1, 687.

Czyzyk, A., Tawecki, J., Sadowski, J., Ponikowska, I. \& SzCZEPANIK, Z. (1968) Effect of biguanides on intestinal absorption of glucose. Diabetes, 17, 492.

Fearnley, G.R. (1969) In discussion. Postgraduate Medical Journal, May Supplement, 19.

Fordtran, J.S., Clodi, P.H., Soergel, K.H. \& IngelFINGER, F.J. (1962) Sugar absorption tests, with special reference to 3-0-Methyl-D-glucose and D-xylose. Annals of Internal Medicine, 57, 883.

FRAYN, K.N. \& ADNITT, P.I. (1971) Estimation of 3-0Methyl-D-glucose in the presence of glucose. Journal of clinical Pathology (In press.)

Goodhart, J.M. \& Kingston, G.R. (1969) Modification of the method for the estimation of xylose in urine. Journal of clinical Pathology, 22, 621.
Hollobaugh, S.L., Rao, M.B. \& Kruger, F.A. (1967) Evidence for significant 'non-peripheral' effects of phenformin (DBI) on glucose metabolism in man. Clinical Research, 15, 428.

HOOPER, B.M. (1967) Diabetes mellitus treated with phenformin. British Journal of Clinical Practice, 21, 389.

Lyngsøe, J. \& Trap-Jensen, J. (1969) Phenformin-induced hypoglycaemia in normal subjects. British Medical Journal, 2, 224.

Mainguet, P., Czyzyk, A., Lauvaux, J.P. \& Franckson, J.R.M. (1970) Inhibition of the intestinal absorption of glucose by phenethylbiguanide in human diabetes. In: 7th Congress of the International diabetes Federation, Buenos Aires. Excerpta Medica, International Congress Series, No. 209, 109.

Morley, G., Dawson, A. \& Marks, V. (1968) Manual and AutoAnalyzer methods for measuring blood glucose using guaiacum and glucose oxidase. Proceedings of the Associa tion of Clinical Biochemists, 5, 42.

Stowers, J.M. \& Bewsher, P.D. (1969) Studies on the mechanism of weight reduction by phenformin. Postgraduate Medical Journal, May Supplement, 13.

University Group Diabetes Program (1970) A study of the effects of hypoglycaemic agents on vascular complications in patients with adult onset diabetes. Diabetes, 19, Supplement 2. 